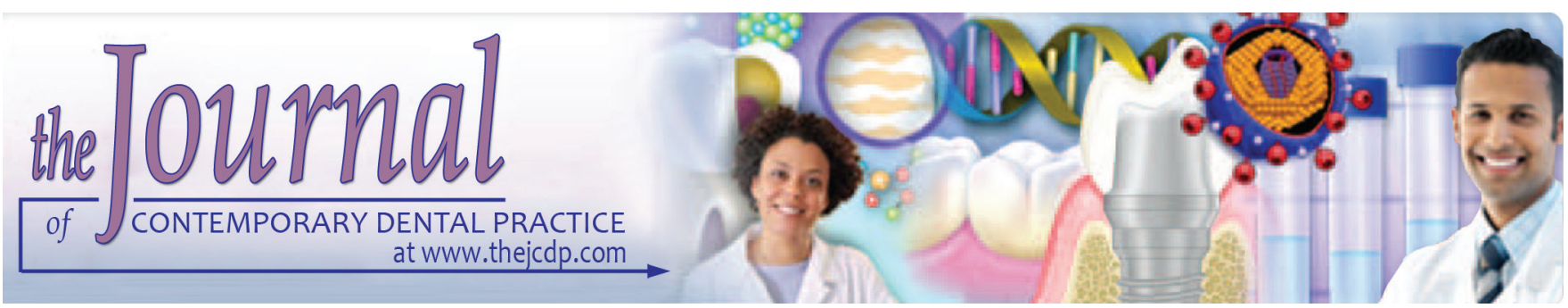

\title{
Triclosan: Hazards of Overuse are not limited to Antibiotics
}

\author{
${ }^{1}$ Nakul Uppal, ${ }^{2}$ Krishnamurthy Bonanthaya
}

How to cite this article: Uppal N, Bonanthaya K. Triclosan: Hazards of Overuse are not limited to Antibiotics. J Contemp Dent Pract 2017;18(11):989-990.

\section{Source of support: Nil}

\section{Conflict of interest: None}

\section{INTRODUCTION}

Antibiotic abuse by patients and overprescription by some dentists are problems in modern healthcare practice. Less understood are the deleterious effects of antiseptics in oral care products marketed for routine home use. Ample literature exists to condemn the routine use of mouthrinses, but criticism focuses on alcohol content and its effects on oral mucosa. ${ }^{1}$ We highlight the more insidious problem of triclosan, which is added as an antiseptic to toothpastes and mouthrinses.

Studies link triclosan and allergic sensitization. ${ }^{2}$ Triclosan is an antimicrobial ingredient in soaps, hand sanitizers, and oral dentifrices but whether the oral cavity requires "disinfection" at all is questionable. A recent advertisement campaign in India for a leading brand of mouthwash claims that "...99.9\% of germs are killed...." However, since a healthy oral cavity is home to approximately 6 billion microorganisms, the wisdom of such compelling marketing can be questioned since even the surviving $0.1 \%$ constitutes a staggeringly high bacterial load. While not disputing the role for antiseptic

${ }^{1}$ Department of Dentistry, All India Institute of Medical Sciences Raipur, Chhattisgarh, India

${ }^{2}$ Department of Oral and Maxillofacial Surgery, Bangalore Institute of Dental Sciences, Bengaluru, Karnataka, India

Corresponding Author: Nakul Uppal, Department of Dentistry All India Institute of Medical Sciences, Raipur, Chhattisgarh India, Phone: +919845628027, e-mail: drnakul@gmail.com mouthrinses after oral surgery, oral hygiene by mechanical plaque control is perfectly adequate for oral health. The threat of Clostridium difficile superinfection following antibiotic misuse leading to pseudomembranous colitis is well-documented and is an ominous warning of the perils of disturbing complex-and not yet fully understood-oral microbiota. There are a vast number and variety of microbial species in the normal oral cavity, and the consequences of disrupting these complex and delicate relationships by topical antiseptics are unknown. A simple example to illustrate the concept of delicate microbial relationships is oral candidiasis that can be a superinfection resulting from overgrowth of endogenous Candida following broad-spectrum antibiotic therapy. It is ironic that disinfection of the mouth is advertised in an era also of probiotic foods and fecal transplants!

Another concern about triclosan is the propensity of the compound to enter the environment via sewage. Lack of degradation of triclosan due to its effect on aquatic bacteria results in bioaccumulation. Maternal-to-fetus transmission of triclosan has been documented as has its presence in breast milk; the hazard to a child is still undetermined. Worryingly, chlorinated tap water reacts with triclosan forming dioxins, which are toxic, as well as endocrinal disruptors. While evidence to avoid use of triclosan in household products is lacking, it is worthy of mention that 2017 onward the US state of Minnesota will ban triclosan from personal care products; one thalidomide disaster is enough. Further, Proctor \& Gamble and Johnson \& Johnson will phase out triclosan from their formulations.

The change required is that, when testing substances, not just toxicity and biocompatibility but also disruption of host microflora needs to be rigorously investigated as standard protocol. In conclusion, it is pertinent to note 
that triclosan was registered with the US Environmental Protection Agency in 1969 as a pesticide. Over a 100 years ago, when Lord Lister advocated soap, water, and common sense as the best tools with which to combat cross-infection, he could not have imagined that the third ingredient of his formula would be in such short supply.

\section{REFERENCES}

1. Gagari E, Kabani S. Adverse effects of mouthwash use. Oral Surg Oral Med Oral Pathol Oral Radiol Endod 1995 Oct;80(4):432-439.

2. Savage JH, Matsui EC, Wood RA, Keet CA. Urinary levels of triclosan and parabens are associated with aeroallergen and food sensitization. J Allergy Clin Immunol 2012 Aug;130(2):453.e7-460.e7. 